\title{
MiR-608 overexpression in idiopathic pulmonary fibrosis (IPF)
}

\author{
Gali Epstein Shochet ${ }^{1,2^{*+}} \oplus$, Lilach Israeli-Shani ${ }^{1,2+}$, Isabelle Kains ${ }^{2}$, Ori Wand ${ }^{1}$ and David Shitrit ${ }^{1,2}$
}

\begin{abstract}
Background: Idiopathic pulmonary fibrosis (IPF) is a chronic progressive disease that causes scarring of the lungs. The disease is associated with the usual interstitial pneumonia pattern, which was not yet fully recapitulated by an animal model. Therefore, the disease is considered 'human specific.' miRNA-608 is a primate specific miRNA with many potential targets, such CdC42 and Interlukin-6 (IL-6) that were previously implicated in IPF pathology.

Objective: To test miR-608 expression and its targets in IPF patient samples.

Methods: RNA was extracted from Formalin fixed paraffin embedded tissue sections ( $N=18)$. miRNA-608 and $C d c 42$ and IL-6 levels were analyzed by qPCR. Acetylcholinesterase (AChE) is another target of miRNA-608. Its' rs I7228616 allele has a single-nucleotide polymorphism causing weakened miR-608 interaction (C2098A). Thus, DNA was extracted from whole blood samples from 56 subjects with fibrosing interstitial lung disease and this region was sequenced for assessment of rs17228616 allele polymorphism.
\end{abstract}

Results: miR-608 is significantly overexpressed in IPF samples in comparison with controls $(p<0.05)$. Cdc42 and IL-6 levels were lower in the IPF patient samples compared with control samples $(p<0.001$ and $p<0.05$, respectively). The frequency of the rs 17228616 minor A-allele was 17/56 (30.4\%) with all patients being heterozygous. This result is significant vs. the published Israeli cohort of healthy individuals, which reported $17 \%$ prevalence of this allele in healthy control volunteers $(p=0.01, \mathrm{OR}=2.1, \mathrm{Cl} 95 \%$ [1.19-3.9]).

Conclusion: miR-608 is overexpressed in IPF patients. While the exact mechanism remains to be discovered, it could potentially promote fibrotic disease.

Keywords: microRNA, IPF, ILD, SNP, CdC42

\section{Introduction}

Idiopathic pulmonary fibrosis (IPF) is a chronic disease that causes scarring of the lungs. The disease is characterized by progressive worsening of dyspnoea and decline in lung function. Many patients with IPF have sub-clinical or clear co-morbid conditions including pulmonary hypertension, gastroesophageal reflux, obstructive sleep

\footnotetext{
*Correspondence: gali.epstein@clalit.org.il

${ }^{\dagger}$ Gali Epstein Shochet and Lilach Israeli-Shani have contributed equally to this work

1 Pulmonary Department, Meir Medical Center, 59 Tchernichovsky St., 44281 Kfar Saba, Israel

Full list of author information is available at the end of the article
}

apnoea, obesity, emphysema, as well as depression and anxiety $[1,2]$.

The disease is limited to the lungs, and is associated with the histopathologic and/or radiologic pattern of usual interstitial pneumonia (UIP). So far, no animal model fully recapitulated the UIP pattern [3] and the disease is considered 'human specific'.

Micro-RNAs (miRNA) are a non-coding RNA sequences, of about 22 nucleotides long that have complementary sequences to particular regions of the mRNA, often in the $3^{\prime} \mathrm{UTR}$, through which regulation occurs. Regulation generally proceeds via either mRNA degradation or inhibition, leading to gene silencing, with the degree of inhibition highly depending on the degree 
of complementarity between the miRNA and its corresponding mRNA target $[4,5]$.

Single-nucleotide polymorphisms (SNPs) are strongly associated with susceptibility to various diseases, including IPF [6-8]. SNPs within miRNA sequences may therefore change properties of the resulting inhibition by altering degree of complementarity $[9,10]$.

miRNA-608 (miR-608, hsa-mir-608) is a long (25 nucleotides) primate specific miRNA. It has many potential targets, such as Rho GTPase CdC42 and Interlukin-6 (IL$6)$, bearing much experimental evidence $[11,12]$. It was previously shown that the miR-608 has differential affinity to the AchE sequence due to C to A change (C2098A) in its $3^{\prime}$-untranslated region (e.g. the minor 'A-allele' and the major ' $\mathrm{C}$-allele', respectively), indicating weakened A-allele AChE-miR-608 interaction [11]. The impaired interaction of the A-allele of AChE with miR-608 predicted weakened $\mathrm{AChE}$ suppression, resulting in elevated levels of free miR-608 molecules to suppress other targets with tight miR-608 binding sites, such as Cdc42 and IL6, which were already shown to be involved in IPF progression $[13,14]$. In this study, we tested miR-608 expression and its targets in human IPF patient samples.

\section{Materials and methods}

\section{RNA purification and quantitative PCR (qPCR)}

Total RNA (including miRNA) was extracted from Formalin fixed paraffin embedded (FFPE) tissue sections taken from 18 IPF patients and 8 control samples, using the miRNeasy FFPE kit (Qaigen, USA) according to the manufacturer's instructions.

mRNA was converted to cDNA using the reverse transcription kit (Applied Biosystems, UK). Reactions were performed with SYBR Green PCR master mix (Applied Biosystems, UK). Primer sequences (purchased from Hylabs, Israel) are listed in Table $1.18 \mathrm{~S}$ served as the reference housekeeping gene. Primers were normalized by specific cDNA standard curves obtained from known amounts of cDNA.

miRNA was converted into cDNA using the qScript microRNA cDNA synthesis kit (Quanta Biosciences, USA) and was then amplified using Perfecta Universal PCR primer kit (Quanta Bioscience, USA). qPCR was performed with Perfecta SYBR Green using specific primers for mir-has-608 (Quanta bioscience, USA). The reference genes (Snord 44 and RNV6) primers were supplied with the kit (sequence not available).

\section{Human blood samples}

Whole blood samples were collected from 56 subjects with fibrosing interstitial lung disease (ILD). DNA was purified from these samples using QaiSymphony (Qaigen). Their demographic data, as well as disease progression and final diagnosis were also recorded.

\section{Gene sequencing}

DNA was amplified using PCRBIO HS Taq master mix (PCR Biosystems) with specific primers for the AchE: Forward 5'-CGCTGGAGCTCCTACATGGT- $3^{\prime}$ and Reverse $5^{\prime}$-ATAGACTCGGCCCCGTGAT-3'. Products were purified using FastAP Thermosensitive Alkaline Phosphatase (Thermo-Fisher scientific). Then, sequencing was performed using BigDye ${ }^{\mathrm{TM}}$ Terminator v3.1 Cycle Sequencing Kit (Thermo-Fisher Scientific) according to manufacturer's instructions. The target sequence was analysed by 3130 Genetic analyser (Applied Biosystems) for determining $\mathrm{A} / \mathrm{C}$ allele single nucleotide polymorphism (SNP) at rs17228616.

\section{Statistical analysis}

Statistical analysis was done using GraphPad Prism version 7.00 for Windows (GraphPad Software, La Jolla California USA, www.graphpad.com). ANOVA was performed to compare differences between multiple cohorts. Student's $t$ tests were employed to analyze differences between two groups. Frequencies were calculated using a Chi-square test. An effect was considered significant when the $p$ value was $<0.05$. All experiments were repeated at least three times.

\section{Ethical approval}

This study was approved by the local Ethics Committee (MMC-18-18). Informed consent was obtained from all subjects.

Table 1 List of primers

\begin{tabular}{lll}
\hline & Forward $\left(\mathbf{5}^{\prime} \mathbf{-} \mathbf{3}^{\prime}\right)$ & Reverse $\mathbf{( \mathbf { 5 } ^ { \prime } \mathbf { - } \mathbf { 3 } ^ { \prime } )}$ \\
\hline CDC42 variant 1 (NM_001791) & CTGTCAAGTATGTGTGGAGTGTTCTG & CTCTTCTTCGGTTCTGGAGGCT \\
CDC42 variant 2 (NM_044472) & TGCACTTACACAGAAAGGCC & CTTCTTCGGTTCTGGAGGCT \\
$18 S$ & AGGAATTGACGGAAGGGCAC & GGACATCTAAGGGCATCACA \\
IL-6 & GGTACATCCTCGACGGCATCT & GTGCCTCTTTGCTGCTTTCAC \\
\hline
\end{tabular}




\section{Results}

MiR-608 is overexpressed in lung tissue samples from IPF patients

The expression of miR-608 in was evaluated in IPF FFPE patient samples $(n=18)$ versus histologically confirmed control tissue samples $(n=8)$. The IPF group consisted of $50 \%$ males, with the average age of $65.5 \pm 8.5$ years. The control group also consisted of $50 \%$ males, with the average age of $59.3 \pm 18$ years. There were no significant differences between the groups regarding comorbidities (other than ILD). Interestingly, a significant overexpression of miR-608 was found in IPF patient samples, in comparison with controls $(p<0.05$, Fig. 1). Using the miRDB search (http://mirdb.org/cgibin/search.cgi), 975 possible targets for miR-608 were identified. These results were then subjected to WEBbased Gene set analysis toolkit (WebGestalt, http:// www.webgestalt.org). We analysed the overrepresentation analysis (ORA) for disease (OMIM) functional database. Interestingly, IPF was the most significantly over-represented disease, with an enrichment ratio of 78 (PULMONARY FIBROSIS, IDIOPATHIC, gene set $178500, \mathrm{FDR}=0.00025)$.

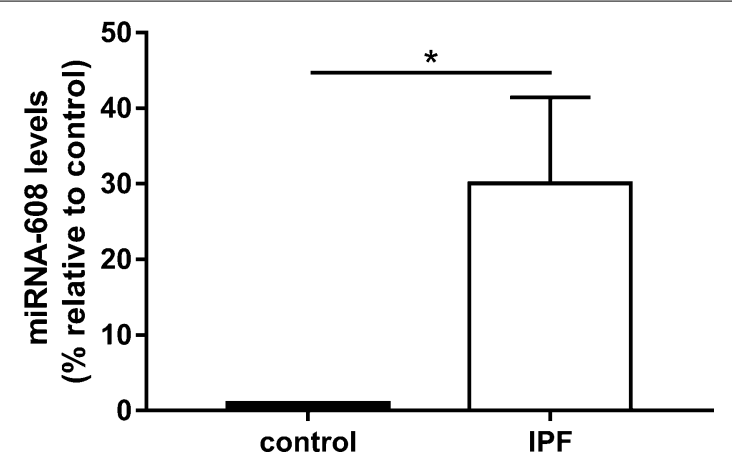

Fig. 1 miR-608 is overexpressed in lung tissue samples from IPF patients. RNA was extracted from IPF and non-IPF (control) FFPE samples. miRNA-608 levels were evaluated by qPCR. Figure shows mean \pm SEM. ${ }^{* * *} p \leq 0.001$, Student's paired $t$ test $(n=18)$

\section{Cdc42 levels are significantly reduced in IPF patient} samples

RhoGTPase Cdc42 is an established target of miR-608 [11]. Moreover, it was recently implicated in lung fibrosis [13]. Thus, we measured the expression level of Cdc42 in IPF patient samples, in comparison to controls. Both Cdc42 transcript variant levels (v1 NM_001791 and v2 NM_044472) were significantly lower in samples from IPF patients compared to the control samples $(p<0.001$, Fig. 2a, b).

\section{IL-6 levels are downregulated in IPF patient samples}

IL-6 is well-known cytokine in IPF and another validated target of miR-608 [11]. qPCR was performed to measure the expression of IL- 6 in IPF patients compared with healthy individuals. In fact, IL- 6 levels were lower in the IPF patient FFPE samples compared with controls $(p<0.05$, Fig. $2 \mathrm{c})$. This result is in key with the hypothesis stating an inhibitory relationship between miR-608 and its targets.

\section{The C2098A substitution (minor rs17228616 allele)} at the $\mathrm{AChE}$ sequence is more prevalent in IPF patients Since both IL-6 and Cdc42 were downregulated in the FFPE samples from patients with IPF, we hypothesized that the presence of the minor A-allele (C2098A) could be higher in subjects with IPF. Thus, 56 patients were recruited and their DNA was sequenced for the A/C allele. Patient characteristics are listed in Table 2. 62.5\% were male with the average age of $65.82 \pm 12$.

Of these subjects, the frequency of the A-allele was $17 / 56$ (30.4\%) with all patients being heterozygous for the minor A-allele (Fig. 3). This result is significant vs. the published Israeli cohort of healthy individuals, which reported $17 \%$ prevalence of this allele in healthy control volunteers ( $p=0.01, \mathrm{OR}=2.1, \mathrm{CI} 95 \%$ [1.19-3.9]).

Of them, 64.5\% were diagnosed with IPF and the rest with other types of ILD [e.g. NSIP (14.5\%), silicosis (3.6\%), hypersensitivity pneumonitis (HP, 3.6\%), sarcoidosis
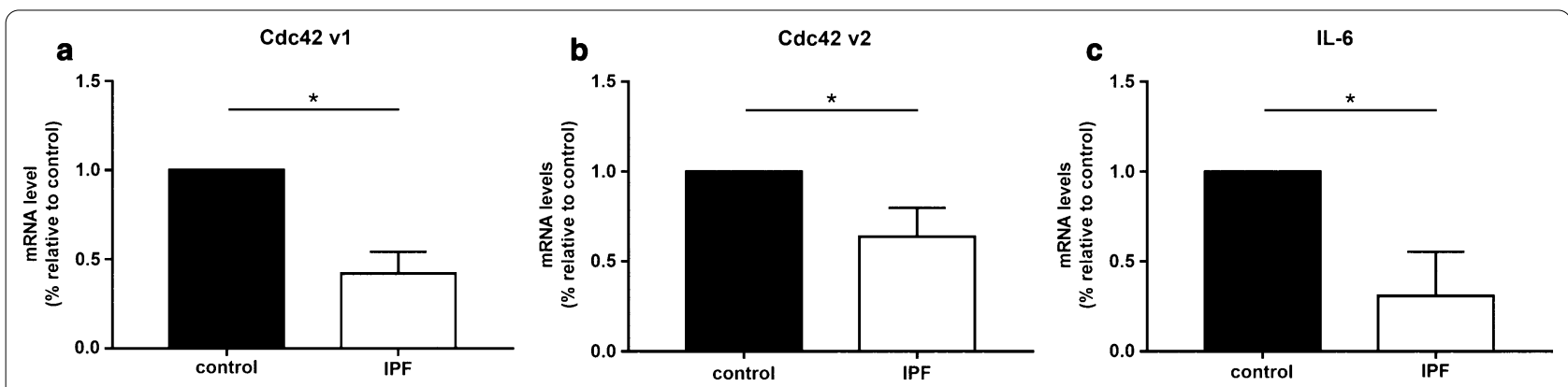

Fig. 2 miR-608 target levels are significantly reduced in IPF patient samples. RNA was extracted from IPF and non-IPF (control) FFPE samples. The two variants of $\operatorname{Cdc} 42(\mathbf{a}, \mathbf{b})$ and IL-6 (c) mRNA levels were evaluated by qPCR. Figures show mean $\pm S E M,{ }^{*} p \leq 0.5$, Student's paired $t$ test $(n=15)$ 
Table 2 Patient characteristics

\begin{tabular}{llll}
\hline Parameter & $\begin{array}{l}\text { A-allele } \\
\mathbf{n = 1 7}\end{array}$ & $\begin{array}{l}\text { C-allele } \\
\mathbf{n = 3 9}\end{array}$ & $\boldsymbol{p}$ value \\
\hline Age & $62 \pm 14$ & $62.4 \pm 11$ & 0.9 \\
Gender (\%male) & $12(70 \%)$ & $23(58.9 \%)$ & 0.41 \\
Smoker & $13(76.4 \%)$ & $20(51.2 \%)$ & 0.08 \\
Rapidly progressing disease ${ }^{a}$ & $6(35.2 \%)$ & $8(20.5 \%)$ & 0.24 \\
FVC \% & $59 \pm 14.5$ & $70 \pm 19$ & 0.02 \\
DLCO\% & $46.2 \pm 17$ & $48.6 \pm 17$ & 0.66 \\
BMI & $27.6 \pm 5$ & $28.4 \pm 4.5$ & 0.61 \\
IHD & $5(29.4 \%)$ & $7(17.9 \%)$ & 0.34 \\
CHF & $1(5.9 \%)$ & $2(5.1 \%)$ & 0.9 \\
Diabetes & $7(41.2 \%)$ & $16(41 \%)$ & 1 \\
Anxiety & $1(5.9 \%)$ & $6(15.4 \%)$ & 0.3 \\
Hypertension & $6(35.2 \%)$ & $11(28.2 \%)$ & 0.64 \\
Osteoporosis & $4(23.5 \%)$ & $7(17.9 \%)$ & 0.63 \\
Malignancy & $1(5.9 \%)$ & $5(12.8 \%)$ & 0.44 \\
\hline
\end{tabular}

$B M I$ body mass index, CHF Congestive Heart Failure, DLCO diffusing capacity for carbon monoxide, FVC forced vital capacity, IHD Ischemic heart disease

a A fall in \%FVC of over $10 \%$ per year was defined as rapidly progressing

(1.8\%) and the rest were determined as unclassifiable pulmonary fibrosis ILD (12\%)]. In addition, we followed up these patients' disease progression to determine whether they are rapidly progressing, as previously defined by the annual decline in FVC\% [15-18]. We found that of the minor allele population, more patients presented with a rapid progressing disease $(35.4 \%$ vs. $20 \%, p=0.24)$, yet this result did not reach significance.

When comparing between the A to the C allele groups' lung function tests at the time of diagnosis, a significantly reduced $\mathrm{FVC}$ at diagnosis for the A group was observed (Table 2, $p=0.02$ ).

\section{Discussion}

Numerous pathways were implicated and major progress has been made towards understanding IPF etiology [19]. However, as recently stated by McDonough et al. [20], since the UIP pattern cannot be replicated in animal models, we have no information about what regulates progression of IPF in the human lung. Thus, in this work we focused on the primate specific miRNA that was previously implicated in aging related diseases. We found that miR-608 was significantly upregulated in IPF tissue samples. In addition, we found that the minor rs17228616 allele was more abundant in IPF patients than in the general population.

miR-608 is located on human chromosome 10q24.31. Although not aforementioned in IPF [21], current studies in tumors indicate that miR-608 affects cell proliferation, invasion, migration and apoptosis [22, 23]. Although the expression level of miR-608 was found to be downregulated in several types of cancer [10, 24-27], these studies did not take into account the SNPs that can significantly affect miRNA stability and function [9].

The major SNP of miR-608 mentioned in cancer is the rs4919510 variant $G$ allele. This SNP was suggested to affect the expression of mature miR-608, as well as that of the proinflammatory cytokines TNF- $\alpha$, IL-6, and IL-1 $\beta$. Nevertheless, there are conflicting results for the association between the presence of miR-608 rs4919510 and susceptibility to tumors [10, 28-30]. Although IPF and lung cancer are sometimes seen in the same patients [31], since we observed an upregulation in the miR-608, rs4919510 was not studied and our focus was shifted to other directions.

To date, limited targets of miR-608 have been confirmed in-vitro, and were mostly performed in tumor cell lines [32]. Our study focused on two targets that
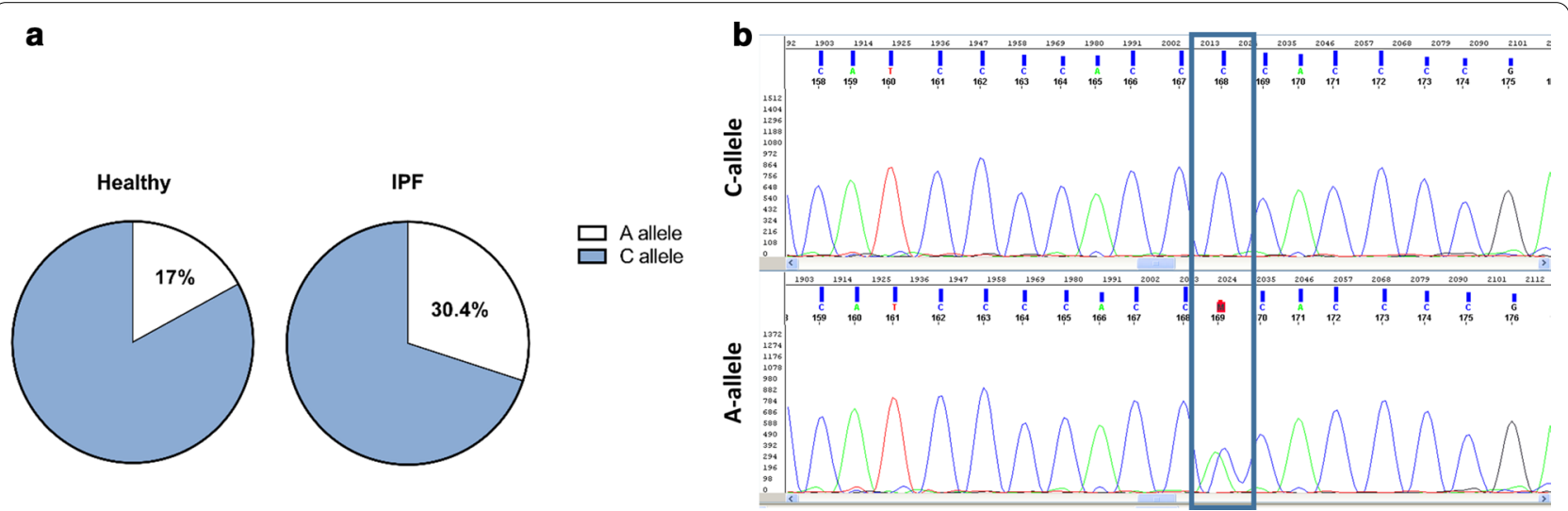

Fig. 3 The C2098A substitution (minor rs17228616 allele) at the AChE sequence is more prevalent in IPF patients. DNA was extracted from 56 subjects with progressive fibrosing ILD. AChE miR-609 target sequence was analysed for determining A/C allele rs 17228616 SNP (a). b is a representative image of sequencing output showing C (top panel) and A heterozygous allele (bottom panel) 
were previously implicated in IPF, IL-6 and Cdc42 [13, 14]. Both targets were found to be down-regulated in IPF patient samples. Supporting these results, our recent work, showed that the IL-6R protein level is also reduced in tissue samples taken from IPF patient biopsies [14]. A recent study by $\mathrm{Wu}$ et al. [13], suggested that Cdc42 is an important post-transcriptional regulator that may play a significant role in the process of inflammation. They found that AT2 cells that lack Cdc42 are not able to differentiate into AT1 cells and, thus, cannot regenerate new alveoli following lung injury. Although IPF is not considered to be an inflammatory disease per se, pro-inflammatory factors, such as IL-6, TNF-alpha and IFN-y were shown to contribute to disease progression [14, 33].

Our work was inspired by a group of researchers who studied miR-608 in the context of anxiety [11]. Several studies reported that symptoms of depression and anxiety are common in patients with IPF. Such studies indicated prevalence of depression ranges from 24.3 to $49.2 \%$, while that of anxiety reaching $60 \%$ in patients with IPF and other ILDs [34-38]. Although a causality of anxiety or depression could be expected, it is possible to assume a genetic predisposition as well. However, the presence of anxiety was not fully addressed in this work, as it relied on electronic patient records and therefore is most likely underestimated or underrecorded [39]. For this issue to be addressed, there is a need for questionnaires, which were not done in this study.

The altered interaction of miR-608 with AChE and the resulting changes, which give rise to a higher ratio of suppression by miR-608 of its other targets, including CdC42 and IL-6, correlate to our results. Hanin et al. showed that young, healthy volunteers with the minor rs17228616 allele showed elevated blood pressure and reduced cortisol, predicting risk of aging-related diseases, such as IPF. Our cohort of patients with progressive fibrosing ILDs, mostly IPF, was shown to include significantly higher prevalence of minor A-allele in comparison to the healthy cohort presented by this group. Since both populations were from Israel, we can also assume similar genetic backgrounds. These results require further investigation in a large cohort to determine the polymorphism in this patient population.

In conclusion, although the number of patients was limited, a significant effect was reached. We found that miR-608 is overexpressed in IPF patients, and that this population includes $30 \%$ of a specific SNP in AChE that was previously implicated as relevant to aging related diseases. These findings require further research in a large study cohort.

\section{Abbreviations}

AchE: Acetylcholine esterase; BMI: Body mass index; CHF: Congestive Heart Failure; DLCO: Diffusing capacity for carbon monoxide; FFPE: Formalin fixed paraffin embedded; FVC: Forced vital capacity; IHD: Ischemic heart disease; IL6: Interlukin-6; ILD: Interstitial lung disease; IPF: Idiopathic pulmonary fibrosis; ORA: Overrepresentation analysis; SNP: Single-nucleotide polymorphisms; UIP: Usual interstitial pneumonia.

\section{Acknowledgements}

The authors thank Ms. Tatiana Epstein for the English editing. We also thank the team at the Molecular genetics laboratory for their help with the sequencing analyses.

\section{Authors' contributions}

GES drafted the manuscript, designed the experiments and analyzed the results, IK performed the experiments and analyzed the results, OW revised it critically for important intellectual content, while DS and LIS contributed to conception and design, drafting the manuscript for important intellectual content and revised the final version. All authors read and approved the final manuscript.

\section{Funding}

No funding was received for this work.

\section{Availability of data and materials}

The datasets generated and/or analyzed during the current study are not publicly available due to patient confidentiality, but are available from the corresponding author on reasonable request.

\section{Ethics approval and consent to participate}

The study was approved by the Ethics Committee of Meir Medical Center. All procedures were performed in accordance with the Declaration of Helsinki. Signed informed consent was obtained from all patients.

\section{Consent for publication}

Not applicable.

\section{Competing interests}

All authors declare no conflict of interest and have consented for publication.

\section{Author details}

${ }^{1}$ Pulmonary Department, Meir Medical Center, 59 Tchernichovsky St., 44281 Kfar Saba, Israel. ${ }^{2}$ Sackler Faculty of Medicine, Tel Aviv University, Tel Aviv, Israel.

Received: 21 October 2020 Accepted: 9 December 2020 Published online: 05 January 2021

\section{References}

1. Oldham JM, Collard HR. Comorbid conditions in idiopathic pulmonary fibrosis: recognition and management. Front Med (Lausanne). 2017:4:123.

2. Raghu G, Collard HR, Egan JJ, Martinez FJ, Behr J, Brown KK, et al. An official ATS/ERS/JRS/ALAT statement: idiopathic pulmonary fibrosis: evidence-based guidelines for diagnosis and management. Am J Respir Crit Care Med. 2011;183(6):788-824.

3. Tashiro J, Rubio GA, Limper AH, Williams K, Elliot SJ, Ninou I, et al. Exploring animal models that resemble idiopathic pulmonary fibrosis. Front Med (Lausanne). 2017:4:118

4. O'Brien J, Hayder H, Zayed Y, Peng C. Overview of microRNA biogenesis, mechanisms of actions, and circulation. Front Endocrinol (Lausanne). 2018;9:402

5. Macfarlane LA, Murphy PR. MicroRNA: biogenesis, function and role in cancer. Curr Genom. 2010;11(7):537-61.

6. Noth I, Zhang Y, Ma SF, Flores C, Barber M, Huang Y, et al. Genetic variants associated with idiopathic pulmonary fibrosis susceptibility and mortality: a genome-wide association study. Lancet Respir Med. 2013;1(4):309-17. 
7. Manichaikul A, Wang XQ, Sun L, Dupuis J, Borczuk AC, Nguyen JN, et al. Genome-wide association study of subclinical interstitial lung disease in MESA. Respir Res. 2017;18(1):97.

8. Ahn MH, Park BL, Lee SH, Park SW, Park JS, Kim DJ, et al. A promoter SNP rs4073T>A in the common allele of the interleukin 8 gene is associated with the development of idiopathic pulmonary fibrosis via the IL-8 protein enhancing mode. Respir Res. 2011;12:73.

9. Duan R, Pak C, Jin P. Single nucleotide polymorphism associated with mature miR-125a alters the processing of pri-miRNA. Hum Mol Genet. 2007;16(9):1124-31.

10. Wang YF, Ao X, Liu Y, Ding D, Jiao WJ, Yu Z, et al. MicroRNA-608 promotes apoptosis in non-small cell lung cancer cells treated with doxorubicin through the inhibition of TFAP4. Front Genet. 2019;10:809.

11. Hanin G, Shenhar-Tsarfaty S, Yayon N, Yau YH, Bennett ER, Sklan EH, et al. Competing targets of microRNA-608 affect anxiety and hypertension. Hum Mol Genet. 2014;23(17):4569-80.

12. Kang JG, Majerciak V, Uldrick TS, Wang X, Kruhlak M, Yarchoan R, et al. Kaposi's sarcoma-associated herpesviral IL-6 and human IL-6 open reading frames contain miRNA binding sites and are subject to cellular miRNA regulation. J Pathol. 2011;225(3):378-89.

13. Wu H, Yu Y, Huang H, Hu Y, Fu S, Wang Z, et al. Progressive pulmonary fibrosis is caused by elevated mechanical tension on alveolar stem cells. Cell. 2020;180(1):107-121e117.

14. Epstein Shochet G, Brook E, Bardenstein-Wald B, Shitrit D. TGF-beta pathway activation by idiopathic pulmonary fibrosis (IPF) fibroblast derived soluble factors is mediated by IL-6 trans-signaling. Respir Res. 2020;21(1):56

15. McCormack FX, King TE Jr, Bucher BL, Nielsen L, Mason RJ. Surfactant protein A predicts survival in idiopathic pulmonary fibrosis. Am J Respir Crit Care Med. 1995;152(2):751-9.

16. Selman M, Carrillo G, Estrada A, Mejia M, Becerril C, Cisneros J, et al. Accelerated variant of idiopathic pulmonary fibrosis: clinical behavior and gene expression pattern. PLoS ONE. 2007;2(5):e482

17. Trujillo G, Meneghin A, Flaherty KR, Sholl LM, Myers JL, Kazerooni EA, et al. TLR9 differentiates rapidly from slowly progressing forms of idiopathic pulmonary fibrosis. Sci Transl Med. 2010;2(57):57ra82.

18. Balestro E, Calabrese F, Turato G, Lunardi F, Bazzan E, Marulli G, et al. Immune inflammation and disease progression in idiopathic pulmonary fibrosis. PLoS ONE. 2016;11(5):e0154516.

19. Lederer DJ, Martinez FJ. Idiopathic pulmonary fibrosis. N Engl J Med. 2018;379(8):797-8.

20. McDonough JE, Ahangari F, Li Q, Jain S, Verleden SE, Herazo-Maya J, et al. Transcriptional regulatory model of fibrosis progression in the human lung. JCI Insight. 2019;4(22):e131597. https://doi.org/10.1172/jci.insig ht.131597.

21. Bagnato G, Roberts WN, Roman J, Gangemi S. A systematic review of overlapping microRNA patterns in systemic sclerosis and idiopathic pulmonary fibrosis. Eur Respir Rev. 2017;26(144):160125. https://doi. org/10.1183/16000617.0125-2016

22. Wang Z, Xue Y, Wang P, Zhu J, Ma J. MiR-608 inhibits the migration and invasion of glioma stem cells by targeting macrophage migration inhibitory factor. Oncol Rep. 2016;35(5):2733-42.

23. Liang Z, Wang $X$, Xu X, Xie B, Ji A, Meng S, et al. MicroRNA-608 inhibits proliferation of bladder cancer via AKT/FOXO3a signaling pathway. Mol Cancer. 2017;16(1):96.

24. Miao Z, Guo X, Tian $L$. The long noncoding RNA NORAD promotes the growth of gastric cancer cells by sponging miR-608. Gene. 2019;687:116-24.
25. Zhang Y, Schiff D, Park D, Abounader R. MicroRNA-608 and microRNA-34a regulate chordoma malignancy by targeting EGFR, BCl-xL and MET. PLoS ONE. 2014;9(3):e91546.

26. Yang PW, Huang YC, Hsieh CY, Hua KT, Huang YT, Chiang TH, et al. Association of miRNA-related genetic polymorphisms and prognosis in patients with esophageal squamous cell carcinoma. Ann Surg Oncol. 2014;21(Suppl 4):S601-609.

27. Yu HX, Wang XM, Han XD, Cao BF. MiR-608 exerts tumor suppressive function in lung adenocarcinoma by directly targeting MIF. Eur Rev Med Pharmacol Sci. 2018;22(15):4908-16.

28. Dai ZM, Kang HF, Zhang WG, Li HB, Zhang SQ, Ma XB, et al. The associations of single nucleotide polymorphisms in miR196a2, miR-499, and miR-608 with breast cancer susceptibility: a STROBE-compliant observational study. Medicine (Baltimore). 2016;95(7):e2826.

29. Pardini B, Rosa F, Naccarati A, Vymetalkova V, Ye Y, Wu X, et al. Polymorphisms in microRNA genes as predictors of clinical outcomes in colorectal cancer patients. Carcinogenesis. 2015;36(1):82-6.

30. Li MP, Hu YD, Hu XL, Zhang YJ, Yang YL, Jiang C, et al. MiRNAs and miRNA polymorphisms modify drug response. Int J Environ Res Public Health. 2016:13(11):1096.

31. Gershman E, Zer A, Pertzov B, Shtraichman O, Shitenberg D, Heching M, et al. Characteristics of lung cancer in idiopathic pulmonary fibrosis with single lung transplant versus non-transplanted patients: a retrospective observational study. BMJ Open Respir Res. 2020;7(1):e000566.

32. Gu W, Wen D, Lu H, Zhang A, Wang H, Du J, et al. MiR-608 exerts antiinflammatory effects by targeting ELANE in monocytes. J Clin Immunol. 2019:40:147-57.

33. Le TT, Karmouty-Quintana H, Melicoff E, Le TT, Weng T, Chen NY, et al. Blockade of IL-6 Trans signaling attenuates pulmonary fibrosis. J Immunol. 2014;193(7):3755-68.

34. Lee YJ, Choi SM, Lee YJ, Cho YJ, Yoon HI, Lee JH, et al. Clinical impact of depression and anxiety in patients with idiopathic pulmonary fibrosis. PLoS ONE. 2017;12(9):e0184300.

35. Akhtar AA, Ali MA, Smith RP. Depression in patients with idiopathic pulmonary fibrosis. Chron Respir Dis. 2013;10(3):127-33.

36. Ryerson CJ, Berkeley J, Carrieri-Kohlman VL, Pantilat SZ, Landefeld CS, Collard HR. Depression and functional status are strongly associated with dyspnea in interstitial lung disease. Chest. 2011;139(3):609-16.

37. Lindell KO, Olshansky E, Song MK, Zullo TG, Gibson KF, Kaminski N, et al. Impact of a disease-management program on symptom burden and health-related quality of life in patients with idiopathic pulmonary fibrosis and their care partners. Heart Lung. 2010;39(4):304-13.

38. Holland AE, Fiore JF Jr, Bell EC, Goh N, Westall G, Symons K, et al. Dyspnoea and comorbidity contribute to anxiety and depression in interstitial lung disease. Respirology. 2014;19(8):1215-21.

39. Walters K, Rait G, Griffin M, Buszewicz M, Nazareth I. Recent trends in the incidence of anxiety diagnoses and symptoms in primary care. PLoS ONE. 2012;7(8):e41670

\section{Publisher's Note}

Springer Nature remains neutral with regard to jurisdictional claims in published maps and institutional affiliations.

Ready to submit your research? Choose BMC and benefit from:

- fast, convenient online submission

- thorough peer review by experienced researchers in your field

- rapid publication on acceptance

- support for research data, including large and complex data types

- gold Open Access which fosters wider collaboration and increased citations

- maximum visibility for your research: over 100M website views per year

At BMC, research is always in progress.

Learn more biomedcentral.com/submissions 